\title{
A User Equilibrium Assignment Flow Model for Multiairport Open Network System
}

\author{
Honghai Zhang, ${ }^{1}$ Qiqian Zhang, ${ }^{2}$ and Lei Yang ${ }^{1}$ \\ ${ }^{1}$ College of Civil Aviation, Nanjing University of Aeronautics \& Astronautics, Nanjing 210016, China \\ ${ }^{2}$ College of Humanities and Social Sciences, Nanjing University of Aeronautics \& Astronautics, Nanjing 210016, China \\ Correspondence should be addressed to Honghai Zhang; jyzhhnuaa@163.com
}

Received 19 January 2015; Accepted 29 March 2015

Academic Editor: Wei (David) Fan

Copyright (c) 2015 Honghai Zhang et al. This is an open access article distributed under the Creative Commons Attribution License, which permits unrestricted use, distribution, and reproduction in any medium, provided the original work is properly cited.

\begin{abstract}
To reduce flight delays and promote fairness in air traffic management, we study the imbalance problem between supply and demand in airport network system from the view of both the system and the users. First, we establish an open multiairport oriented network flow system with the correlation between the arrival and departure in capacity-constrained airports, as well as the relevance between multiairports united flights. Then, based on the efficiency rationing principle, we propose an optimization model to reassign flow with user equilibrium constraints. These constraints include Gini coefficient, system capacity, and united flights. The model minimizes the total flight delay cost of capacity-constrained airports in the network system. We also introduce some evaluation indexes to quantitatively analyze fairness among airlines. Finally, with an open multiairport network system and its actual flights data in China, the model is verified. Test results show that the model can be used to coordinate and optimize matching the flow and capacity in the multiairport, make full use of the capacity of airports, and minimize the system delays. The findings can provide a powerful reference of developing scientific and rational assignment strategy for air traffic controllers.
\end{abstract}

\section{Introduction}

It is clear that the phenomenon of growing traffic demand should be met by a concomitant improvement in airport capacity. The long-term programs for increasing airport capacity include construction of new airports and expansion of runway systems at existing airports; the short-term programs consider new operational methods in air traffic flow management (ATFM) and capacity utilization as potentially effective measures for improving the existing capacity resources [1,2]. Analysis shows that optimization of the present airport system by the operational and technological measures might result in increasing current traffic flow by up to $50 \%$ [3]. ATFM aims to optimize flow distribution, improve resource utilization, and promote safety in air traffic by rationalizing the traffic demand and adjusting traffic flow, whose essential purpose is to adjust the air traffic demand and supply with coordinated manner [4]. With the rapid development of air transport, the contradiction between the dramatic increase of air traffic demand and the relative decrease of capacity supply is becoming increasingly prominent, and the air traffic congestion is getting worse. Airports are air traffic management bottlenecks, studying the imbalances between supply and demand of airport traffic has an important realization meaning in solving air traffic congestion, reducing flight delays, and promoting the safety of air traffic. The issue of imbalance between airports traffic demand and supply is mainly that the airports traffic demand exceeds the supply of airports capacity. It belongs to strategic and pretactical air traffic flow management issue. The essence is the coordination configuration between flow and capacity.

Air traffic flow allocation problem has been a growing concern on scholars home and abroad. In order to assign capacity and allocate flow, Gilbo, Ma, Chen, and others have, respectively, established a kind of optimization model to minimize delayed flights quantity or losses in arrival and departure queue. Gilbo presented a model which first considers the runways and arrival and departure fixes jointly as 
a single system resource and secondly considers arrivals and departures simultaneously as two interdependent processes [4]. The model takes into account the interaction between runway capacity and capacities of fixes to optimize the traffic flow through the airport system. The effects are achieved by dynamic time-dependent allocation of airport capacity and flows between arrivals and departures coordinated with the operational constraints at runways and arrival and departure fixes as well as dynamics of traffic demand and weather. $\mathrm{Ma}$ et al. proposed a model for traffic flow allocation and optimal scheduling in the terminal area [5]. The model provides more efficient utilization of existing terminal capacity in each time interval to optimize flow distributions and alleviate consequences of flight delays. The model treats the arrivals and departures as two interdependent processes to optimize the airport flow distribution. The model also considers the dynamic characteristics of the time-dependent allocation of airport capacity and the tradeoff between arrivals and departures along with the dynamic characteristics of traffic demand and weather. Chen et al. set up a multiobjective model to optimize the airside capacity utilization and the traffic flow distribution, which maximize flow through runway and arrival/departure fix by introducing utilization satisfaction level [6]. The result indicates that the proposed model is more effective to decrease overall flight demand queues than Gilbo model results. Within the considered time period, all flight demands are satisfied without any delay at the end of the last $15 \mathrm{~min}$ interval by optimal allocation of flow for each arrival/demand fix of airside. Thus, it guarantees the capacity utilization satisfaction level and gives an effective utilization of airport airside capacity and reduces flight delays. Dell'Olmo and Lulli studied the problem of finding the optimal tradeoff between the number of arrivals and the number of departures in order to reduce a delay function of all the flights [7], using a more realistic representation of the airport capacity, that is, the capacity envelope. Under the assumption of piecewise linear convex capacity envelopes and of the exact interpolation of all the pareto-optimal operational points, they show that the problem can be formulated as a linear programming model. For general airport capacity envelopes, they proposed a dynamic programming formulation with a corresponding backward solution algorithm, which was robust and easy to implement and had a linear computational complexity.

With the development of the collaborative decisionmaking (CDM) in ATFM, collaborative optimization problem emerged. Under collaborative optimization, arrivals and departures are considered jointly and are treated as interdependent operations. When arrival capacity can be traded for departure capacity, collaborative optimization provides the best allocation of capacity to arrivals and departures to maximize the airport throughput and minimize delays. Furthermore, collaborative optimization uses flight priorities from airlines and other users in the optimization model. Dell'Olmo and Lulli presented a collaborative optimization model taking into account user priorities (preserving integrity of banks at hub airports), which can be used as a decision support tool for solving ATFM problems at airports [7]. During years 2008 and 2010, Zhang and Hu took into account airport capacity dynamic constraints, tradeoff between arrival and departure, or/and the interaction between multiple airports and, respectively, established some collaborative optimization models of capacity utilization and flow assignment for single and multiple airports terminal area [8-11]. To find optimal strategy, these models minimized the total flights' weighted delay costs and introduced delay cost coefficients for each flight as airline's decision-preferential information. The results of some experiment have shown that these proposed models can not only have a balance between traffic flows and capabilities and make best use of system resource but also minimize delay cost with airline's preferences.

Researches above are achieved by optimizing by flight line-up or with flight delay coefficient taking into account users' expection or preference information. They make the issue more reasonable to some degrees but have not considered the fairness among users, that is, the delay degree of influence on the airlines, which is caused from the imbalance between supply and demand. What is more, they have not considered the spread phenomenon of delay from the system's view. In this view, from both system optimization and user equilibrium perspectives, we research the issue of imbalance between demand and supply in an open airport network system, so as to find out a tradeoff among the balancing users' needs, optimizing the system's flow distribution and making full use of the system's capacity.

\section{Open Multiairport Network System}

An open multiairport network system is a kind of air traffic network system in which some airports are known but the others are unknown, referring to Figure 1. The system considers not only the flights connecting among the airports, but also the flights turnover within a specific airport. These two elements make up the air traffic flow within the network system. An airport in the system is shown by a model which consists of points A, B, C, D, and E. Under normal circumstances, the arrival flights from other airports enter into point $\mathrm{A}$; then, some flights enter into point $\mathrm{B}$ ending their journeys; the other continuous flights turn over after point $\mathrm{C}$ and turn into departure flights with the flights from point $\mathrm{D}$. The self-loop arc at point $\mathrm{C}$ indicates the turnover; the selfloop arc at point D stands for the delay in Figure 1.

An open multiairport network system flow is made up by these airports' flow. An airport flow consists of the arrival and the departure flow. The capacity of the network system is mainly reflected in the capacity of each airport, including arrival and departure capacity. Since the arrival and departure of the flights are closely related and interacting with each other, airport capacity also significantly changes with the weather conditions and is even directly related to the type of aircrafts, the ratio of combination, and the order of takeoff and landing. Researches have shown that the arrival and departure capacity can usually be displayed as the airport capacity curve figure as Figure 2 shows, which varies under different weather conditions $[12,13]$. 


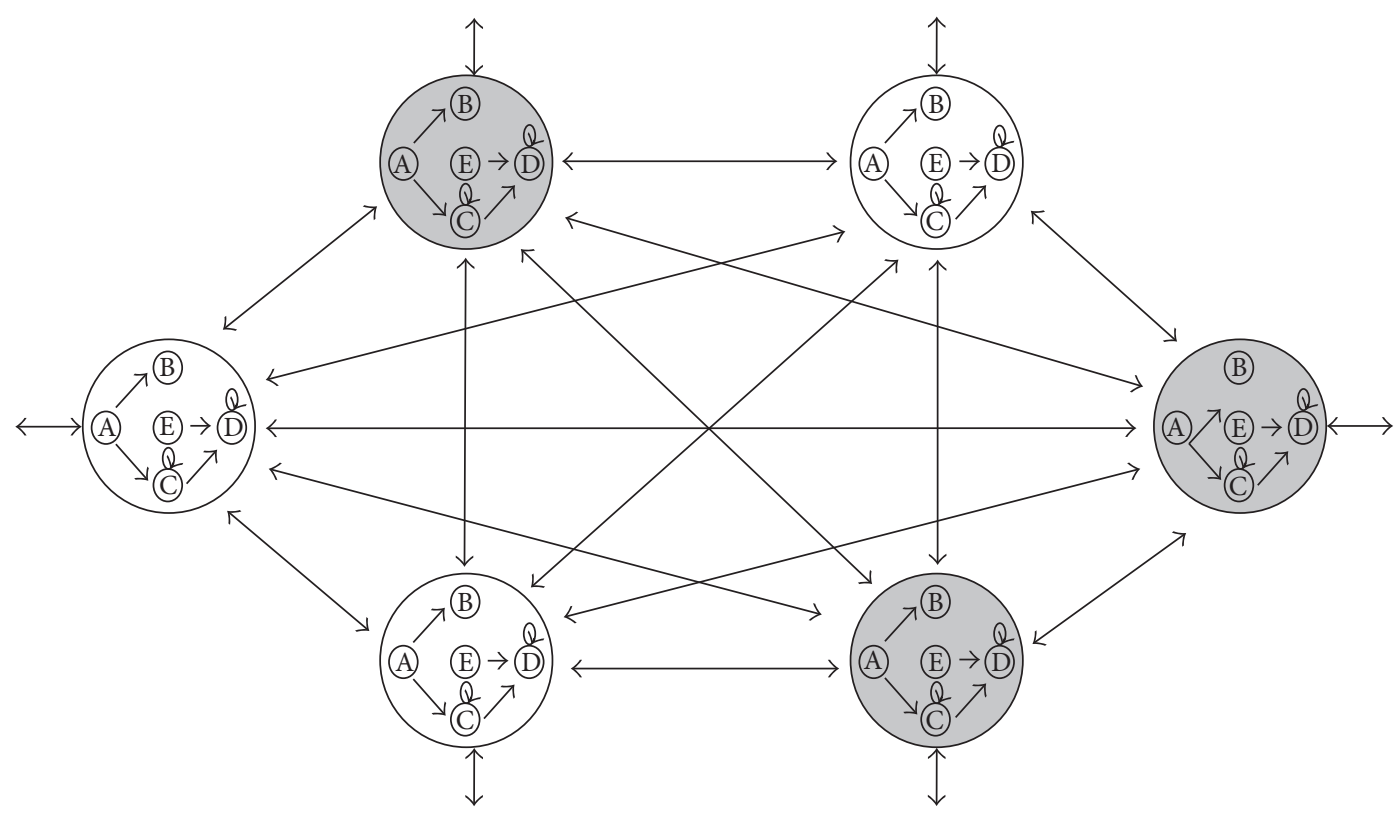

FIGURE 1: An open multiairport network system.

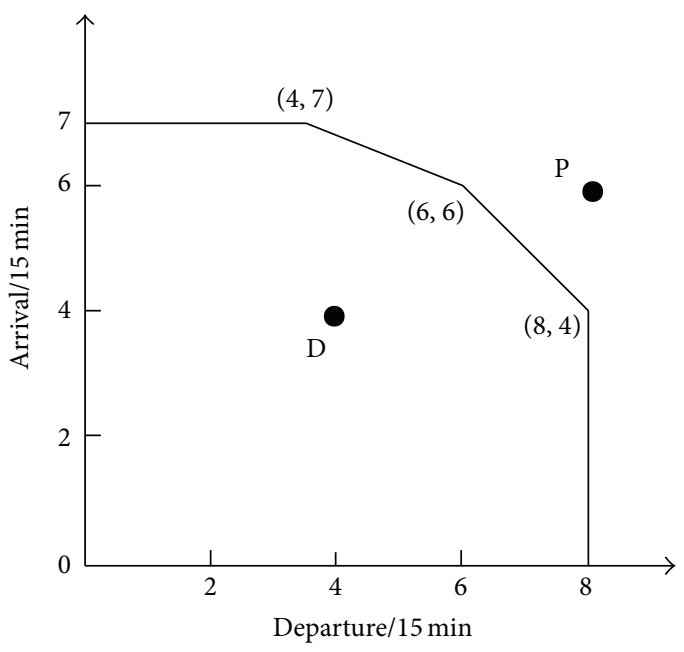

FIGURE 2: An airport capacity curve.

The vertical axis indicates the number of arrival flights every 15 minutes and the horizontal axis is the number of departure flights every 15 minutes. If the traffic demand exceeds the capacity limits, such as point $\mathrm{P}$ in Figure 2, delay is inevitable. In order to minimize the delay, a Pareto optimistic capacity configuration point (or flow distribution point) on or inside the curve should be found to rearrange the traffic flow and make the best use of capacity. As a result, in the airport network system, if a certain airport flow exceeds its capacity (as the grey airport shown in Figure 1), some airports in the system will be affected and the related flights will be delayed.

\section{A User Equilibrium Assignment Model}

3.1. Problem Description. The user equilibrium assignment problem for the open airport network system can be described as follows: given certain air traffic demand and capacity supply at each airport within this system in a certain period of time, how to reasonably rearranging these airport's demand and capacities is the key to minimize the total delay loss among the interests of users fairly when some airports exceed their limits. The basic clue of modeling is to divide the time period into several time slots and deploy the best arriving/departure periods for the flights in capacityconstrained airports according to the principles of users equally delayed to rearrange the demands, so as to make these airports' traffic flow and capacity harmonious in all these time slots. The model requires four hypotheses.

(1) During a given period, the capacity curves of the capacity-constrained airports under different weather conditions are known in the network system.

(2) The air traffic demand not accommodated in a given slot can be always arranged in the first slot outside of the period whose capacity has no upper limits, which assures that the problem can be solved.

(3) During the given period, the capacities of the other airports in the network system have no upper limits.

(4) All the flights cannot take off or arrive before their scheduling time and cannot be delayed indefinitely, which is rational in real life. 
3.2. Symbol Definition. T, the time interval of preassignment, is composed by a number of consecutive time segments $t$ whose length is $\Delta$ (usually $15 \mathrm{~min}$ ), where

$$
T=\left\{t_{1}, t_{2}, \ldots, t_{i}, \ldots, t_{N}\right\}, \quad t \in T .
$$

Define $t_{N+1}$ as the next time segment which is out of the time interval and its capacity is infinite.

$A$ is the set of airlines involved in the capacity-constrained airports in the interval $T, a \in A$.

$I$ is the set of the capacity-constrained airports which are in the multiairport open network, $i \in I$.

$F$ is the set of flights departing from airport $i$ and arriving at airport $j$ which are both in the network system, where $i, j \in$ $I, f \in F$.

$F_{i}$ is the set of flights in the capacity-constrained airport $i$, where $i \in I, F_{i} \subset F . F_{i}$ is composed of the set of departure flights $\operatorname{Dep}_{i}$ and the set of arrival flights $\operatorname{Arr}_{i}$, where

$$
\begin{aligned}
F_{i} & =\operatorname{Dep}_{i} \cup \operatorname{Arr}_{i}, \\
\operatorname{Dep}_{i} & =\operatorname{Dep}_{i}^{\text {ext }} \cup \operatorname{Dep}_{i}^{\text {int }},
\end{aligned}
$$

where $\operatorname{Dep}_{i}^{\text {ext }}$ is the set of flights that depart from the capacityconstrained airport $i$ and arrive at non-capacity-constrained airports. Dep ${ }_{i}^{\text {int }}$ is the set of flights that depart from the capacity-constrained airport $i$ and arrive at the capacityconstrained airport $j$ :

$$
\operatorname{Arr}_{i}=\operatorname{Arr}_{i}^{\text {ext }} \cup \operatorname{Arr}_{i}^{\text {int }}
$$

where $\operatorname{Arr}_{i}$ is the set of flights that depart from the capacityconstrained airport $i$ and arrive at non-capacity-constrained airports and $\mathrm{Arr}_{i}^{\text {int }}$ is the set of flights that depart from the capacity-constrained airport $j$ and arrive at the capacityconstrained airport $i$.

$F_{a}$ is the set of flights for airline $a$, where

$$
F_{a}=\{f \in F \mid O(f)=a\}, \quad F_{a} \subseteq F, O: F \longrightarrow A .
$$

It means the matching relationship between flights and airline, where

$$
F_{a}=\bigcup_{i \in I} F_{i, a}
$$

$F_{i, a}$ is the flights set of airline $a$ at capacity-constrained airport, where

$$
F_{i, a}=\operatorname{Dep}_{i, a}^{\text {ext }} \cup \operatorname{Dep}_{i, a}^{\text {int }} \cup \operatorname{Arr}_{i, a}^{\text {ext }} \cup \operatorname{Arr}_{i, a}^{\text {int }} .
$$

$\operatorname{Dep}_{i, a}^{\text {ext }}$ is the set of flights of airline $a$ that depart from the capacity-constrained airport $i$ and arrive at the non-capacityconstrained airport and Dep int is the set of flights of airline $a$ that depart from the capacity-constrained airport $i$ and arrive at the capacity-constrained airport $j$. Arr $\mathrm{Art}_{i, a}^{\mathrm{ext}}$ is the set of flights of airline $a$ that depart from the non-capacity-constrained airport and arrive at the capacity-constrained airport $i$, and Arr $_{i, a}^{\text {int }}$ is the set of flights of airline $a$ that depart from the capacity-constrained airport $j$ and arrive at the capacityconstrained airport $i$.
$X$ is the set of connecting flights that have continuous range in $I$, where

$$
X=\left\{\left(f, f^{\prime}\right) \mid f, f^{\prime} \in F\right\} .
$$

$f$ is proceeding flight, and $f^{\prime}$ is subsequent flight.

$\Delta t_{f, f^{\prime}}$ is the minimum time interval between $f$ and $f^{\prime}$ in individual capacity-constrained airports, where

$$
\left(f, f^{\prime}\right) \in X
$$

$e_{f}$ is the estimated time of arrival or departure of flight $f$, where $e_{f}^{d}$ is the estimated takeoff time and $e_{f}^{a}$ is the estimated landing time.

$T_{f}$ is the set of probable departure time segment or land time segment for the flight $f$, where

$$
T_{f}=\left\{t \in T \mid t \geq e_{f}\right\} \cup\left\{t_{N+1}\right\} \text {. }
$$

$\psi_{t}^{i}(u, v)$ is the arrival and departure capacity curve of airport $i$ in segment $t$, where $t \in T, i \in I$.

$U_{t}^{i}$ is the maximum arrival capacity of airport $i$ in segment $t$.

$V_{t}^{i}$ is the maximum departure capacity of airport $i$ in segment $t$.

$u_{t}^{i}$ is the arrival flow of airport $i$ in segment $t$, where

$$
u_{t}^{i}=\sum_{f \in \operatorname{Arr}_{i}} y_{f}(t), \quad i \in I, t \in T .
$$

$v_{t}^{i}$ is the departure flow of airport $i$ in segment $t$, where

$$
v_{t}^{i}=\sum_{f \in \operatorname{Dep}_{i}} x_{f}(t), \quad i \in I, t \in T .
$$

The decision variables are

$$
\begin{aligned}
& x_{f}(t)= \begin{cases}1, & \text { if flight } f \text { takes off at time segment } t, \\
0, & \text { otherwise, }\end{cases} \\
& y_{f}(t)= \begin{cases}1, & \text { if flight } f \text { lands at time segment } t, \\
0, & \text { otherwise. }\end{cases}
\end{aligned}
$$

3.3. Mathematical Model. The objective function of the model is to minimize the total delay costs of flights in capacity-constrained airports of the open network system, including the delay costs of flights to take off from and land at capacity-constrained airports, flights to take off from capacity-constrained airport and land at capacityunconstrained airports, and flights to take off from capacityunconstrained airport and land at capacity-constrained airports. In order to avoid double counting the delay in the multiairport network system, the delay costs of flights to take off from and land at capacity-constrained airports are represented in departure delay; the delay costs of flights 
to take off from capacity-constrained airport and land at capacity-unconstrained airport are represented in departure delay; the delay costs of flights to take off from capacityunconstrained airport and land at capacity-constrained airport are represented in arrival delay, as shown in the following formulation:

$$
\begin{aligned}
\min \sum_{i \in I}\left\{\sum_{f \in \operatorname{Dep}_{i}^{\mathrm{int}}} \sum_{t \in T_{f}}\left(t-e_{f}^{d}\right) \cdot x_{f}(t)\right. \\
+\sum_{f \in \operatorname{Dep}_{i}^{\mathrm{ext}}} \sum_{t \in T_{f}}\left(t-e_{f}^{d}\right) \cdot x_{f}(t) \\
\left.+\sum_{f \in \operatorname{Arr}_{f}^{\mathrm{ext}}} \sum_{t \in T_{f}}\left(t-e_{f}^{a}\right) \cdot y_{f}(t)\right\} .
\end{aligned}
$$

Then,

$$
\begin{aligned}
\min \sum_{i \in I}\left\{\sum_{f \in \mathrm{Dep}_{i}} \sum_{t \in T_{f}}\left(t-e_{f}^{d}\right) \cdot x_{f}(t)\right. \\
\left.+\sum_{f \in \operatorname{Arr}_{f}^{\mathrm{ext}}} \sum_{t \in T_{f}}\left(t-e_{f}^{a}\right) \cdot y_{f}(t)\right\}
\end{aligned}
$$

and is subject to the following constraints.

(1) Segment assignment constraint: it is used to ensure that each flight has only one departure/arrival time segment in the set of takeoff/landing time segments. Among them, formulation (15) is the constraint of departure time segment assignment and formulation (16) is the constraint of arrival time segment:

$$
\begin{aligned}
& \sum_{t \in T_{f}} x_{f}(t)=1, \quad x_{f}(t)=\{0,1\}, \forall f \in \operatorname{Dep}_{i}, \forall i \in I, \\
& \sum_{t \in T_{f}} y_{f}(t)=1, \quad y_{f}(t)=\{0,1\}, \forall f \in \operatorname{Arr}_{i}, \forall i \in I .
\end{aligned}
$$

(2) Capacity-constrained constraint: it constitutes the flow distribution point field of capacity-constrained airport (within or on the capacity curve), finding optimal distribution point in the point region to ensure that arrival and departure flows match its capacity, which is a tradeoff between arrival and departure. Among them, $\alpha_{t}^{i}, \beta_{t}^{i}$, and $\gamma_{t}^{i}$ are the coefficients of the capacity curve $\psi_{t}^{i}(u, v)$. As shown in Figure 2, $0 \leq u_{t} \leq 7,0 \leq v_{t} \leq 8, u_{t}+v_{t} \leq 12$, and $2 u_{t}+$ $v_{t} \leq 18$ constitute the flow distribution point field. Among them, formulation (17) is the largest capacity constraints; formulation (18) is the capacity balance constraints:

$$
\begin{gathered}
0 \leq u_{t}^{i} \leq U_{t}^{i}, \quad 0 \leq v_{t}^{i} \leq V_{t}^{i}, \quad \forall t \in T, \quad \forall i \in I, \\
\alpha_{t}^{i} \cdot u_{t}^{i}+\beta_{t}^{i} \cdot v_{t}^{i} \leq \gamma_{t}^{i}, \quad \forall t \in T, \quad \forall i \in I .
\end{gathered}
$$

(3) Continuous flight constraint: it is used to ensure connecting flights have enough time till turnaround:

$$
t_{f}-t_{f^{\prime}} \geq \Delta t_{f, f^{\prime}}, \quad \forall\left(f, f^{\prime}\right) \in X .
$$

(4) Airborne hour constraint: it is used to meet estimated airborne hours of flights between capacity-constrained airports unchanged after optimization. As the goal of optimization is to get the flow of each time segment, the constraints are shown as in this equation to ensure the model has a feasible solution. However, the optimization times of arrival and departure and estimated airborne of these flights still meet equivalent relationships:

$$
\begin{aligned}
& 0 \leq\left(t_{f_{i}}-t_{f_{j}}\right)-\left(e_{f_{i}}-e_{f_{j}}\right) \leq \Delta \\
& f_{i} \in \operatorname{Arr}_{i}, \quad f_{j} \in \operatorname{Dep}_{j} ; \quad i, j \in I .
\end{aligned}
$$

(5) User equilibrium constraint: it is used to ensure the equal distribution of delay among different airlines. This paper uses the Gini coefficient which reflects the fairness to quantify the equilibrium and establish the constraint. Gini coefficient reflects the extent of unfairness in resources distribution problem; its value varies from 0 to 1 . It indicates that the lower the number, the more equal the distribution. For the problem of this paper, the Gini coefficient $G$ can be expressed as (21), and its practical significance is the deviation of average delay between airlines. The lower the number, the smaller the loss deviation, and it indicates that the distribution tends toward more balance and fairness:

$$
G=\frac{\sum_{l=1}^{m-1} \sum_{l<k \leq m}\left|q_{l} C_{k}-q_{k} C_{l}\right|}{\sum_{a \in \text { Air }} C_{a}}
$$

where $C_{a}$ is the delay loss of airline $a$, defined as the following formulation:

$$
C_{a}=\sum_{i \in I}\left\{\sum_{f \in \operatorname{Dep}_{i, a}} \sum_{t \in T_{f}}\left(t-e_{f}^{d}\right)+\sum_{f \in \operatorname{Arr}_{i, a}^{\text {ext }}} \sum_{t \in T_{f}}\left(t-e_{f}^{a}\right)\right\} .
$$

$q_{a}$ is flight proportion of airline $a$. It indicates that flow is allotted according to airlines' market share, but not absolute egalitarianism. So it will be more reasonable:

$$
q_{a}=\frac{\left|F_{a}\right|}{|F|}
$$

Then, set $G$ does not exceed a certain small parameter value $\delta$ to limit deviations, $0 \leq \delta \leq 1$ order $G \leq \delta$, and then establish fairness constraints:

$$
\sum_{l=1}^{r-1} \sum_{l<k \leq r}\left|q_{l} C_{k}-q_{k} C_{l}\right| \leq \delta \sum_{a \in A} C_{a}
$$

Define

$$
\begin{aligned}
& d_{k l}^{+}=\frac{\left(\left|q_{l} C_{k}-q_{k} C_{l}\right|+q_{l} C_{k}-q_{k} C_{l}\right)}{2}, \\
& d_{k l}^{-}=\frac{\left(\left|q_{l} C_{k}-q_{k} C_{l}\right|-q_{l} C_{k}+q_{k} C_{l}\right)}{2} .
\end{aligned}
$$


TABLE 1: The flow distribution in the three airports before optimization.

\begin{tabular}{|c|c|c|c|c|c|c|c|c|c|}
\hline \multirow{2}{*}{ Time } & \multicolumn{3}{|c|}{ Beijing Capital Airport } & \multicolumn{3}{|c|}{ Shanghai PuDong Airport } & \multicolumn{3}{|c|}{ Guangzhou BaiYun Airport } \\
\hline & Dep. & Arr. & Total & Dep. & Arr. & Total & Dep. & Arr. & Total \\
\hline $8: 00-8: 14$ & 15 & 0 & 15 & 12 & 0 & 12 & 16 & 1 & 17 \\
\hline $8: 15-8: 29$ & 16 & 1 & 17 & 17 & 0 & 17 & 12 & 3 & 15 \\
\hline $8: 30-8: 44$ & 16 & 3 & 19 & 14 & 6 & 20 & 9 & 1 & 10 \\
\hline $8: 45-8: 59$ & 15 & 10 & 25 & 14 & 4 & 18 & 8 & 5 & 13 \\
\hline 9:00-9:14 & 7 & 3 & 10 & 26 & 3 & 29 & 11 & 3 & 14 \\
\hline $9: 15-9: 29$ & 5 & 2 & 7 & 12 & 5 & 17 & 4 & 5 & 9 \\
\hline $9: 30-9: 44$ & 10 & 13 & 23 & 4 & 5 & 9 & 2 & 7 & 9 \\
\hline $9: 45-9: 59$ & 8 & 19 & 27 & 12 & 39 & 51 & 11 & 8 & 19 \\
\hline 10:00-10:14 & 5 & 2 & 7 & 14 & 9 & 23 & 8 & 4 & 12 \\
\hline $10: 15-10: 29$ & 1 & 5 & 6 & 6 & 28 & 34 & 3 & 4 & 7 \\
\hline 10:30-10:44 & 17 & 11 & 28 & 6 & 13 & 19 & 10 & 3 & 13 \\
\hline $10: 45-10: 59$ & 16 & 22 & 38 & 7 & 18 & 25 & 12 & 13 & 25 \\
\hline 11:00-11:14 & 9 & 7 & 16 & 32 & 9 & 41 & 7 & 3 & 10 \\
\hline $11: 15-11: 29$ & 3 & 11 & 14 & 5 & 16 & 21 & 3 & 6 & 9 \\
\hline 11:30-11:44 & 10 & 7 & 17 & 13 & 9 & 22 & 9 & 5 & 14 \\
\hline 11:45-12:00 & 21 & 20 & 41 & 12 & 11 & 23 & 12 & 18 & 30 \\
\hline Total & 174 & 136 & 310 & 206 & 175 & 381 & 137 & 89 & 226 \\
\hline
\end{tabular}

Then,

$$
\begin{gathered}
d_{k l}^{+}-d_{k l}^{-}=q_{l} C_{k}-q_{k} C_{l} \\
l=1,2, \ldots, r-1 ; \quad l<k \leq r ; \quad d_{k l}^{+}, d_{k l}^{-} \geq 0 .
\end{gathered}
$$

Then, constraints (24) can be turned into the following linear form:

$$
\begin{array}{r}
\sum_{l=1}^{r-1} \sum_{k>l}\left(d_{k l}^{+}+d_{k l}^{-}\right) \leq \delta \sum_{a \in A} C_{a} \\
d_{k l}^{+}-d_{k l}^{-}-q_{l} C_{k}+q_{k} C_{l}=0 \\
l=1,2, \ldots, r-1, \quad l<k \leq r .
\end{array}
$$

\section{User Equilibrium Evaluation Indexes}

The essence of user equilibrium assignment is that airlines share the total delay loss according to the proportion of their flights. Therefore, the pros and cons of equilibrium assignment are mainly a response to total delay loss (i.e., effectiveness) and fairness among airlines. So there are two $e$ evaluation criteria. One is effectiveness criteria: minimize the total delay loss of all flights; the assignment with less total loss is better; otherwise it is more undesirable. The other is fairness criteria: balance the total loss delays among airlines as much as possible. Overall evaluation index can be reflected by means of flight delay deviation, that is, Gini coefficient. The smaller the delay deviation between airlines, the more balanced the delay distribution; otherwise, it is more uneven.
To quantitatively evaluate the fairness of a specific airline, an effectiveness-fair degree is used, which is defined as the ratio of airline's total loss proportion and its flight proportion. According to the principle of distribution, the loss proportion airline bears should be equal to its flight proportion. So the effectiveness fair degree of the airline which is close to 1 indicates a fair loss degree borne by the flight proportion; that is, the loss of the airline is more fair; on the contrary, the loss is more unfair. The effectiveness-fair degree of airline $a$ is expressed as the following formulation:

$$
e_{a}=\frac{C_{a} / \sum_{a \in A} C_{a}}{\left|F_{a}\right| /|F|}
$$

\section{Sample Simulation Analysis}

5.1. Simulation Case Study. An open multiairport network system is constituted with some capacity-constrained airports, that is, Beijing Capital Airport, Shanghai PuDong Airport, and Guangzhou BaiYun Airport. Using a typical time interval (8:00-12:00, $N=16, \Delta=15 \mathrm{~min})$ of the actual flight data on a certain day in these three airports, the initial traffic demand distribution in three airports is shown in Table 1. The capacity curves supposed for the three airports and their match charts between demand and capacity in different periods are shown in Figure 3. Apparently, due to capacity constraints, supply and demand are imbalanced in the three major airports during some time segments. The demand of arrival and departure exceeds the capacity limit in some periods (the demand point is beyond the capacity curve in the 
- $(12,39)$

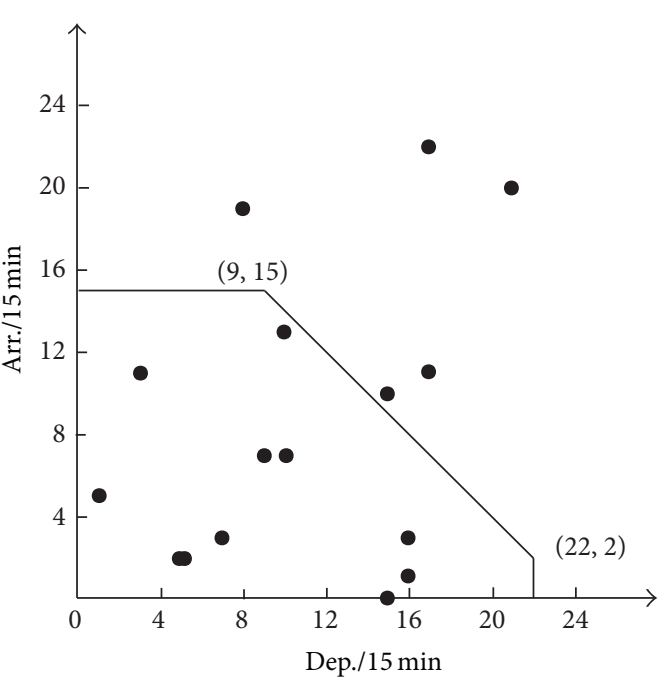

(a) Beijing Capital Airport

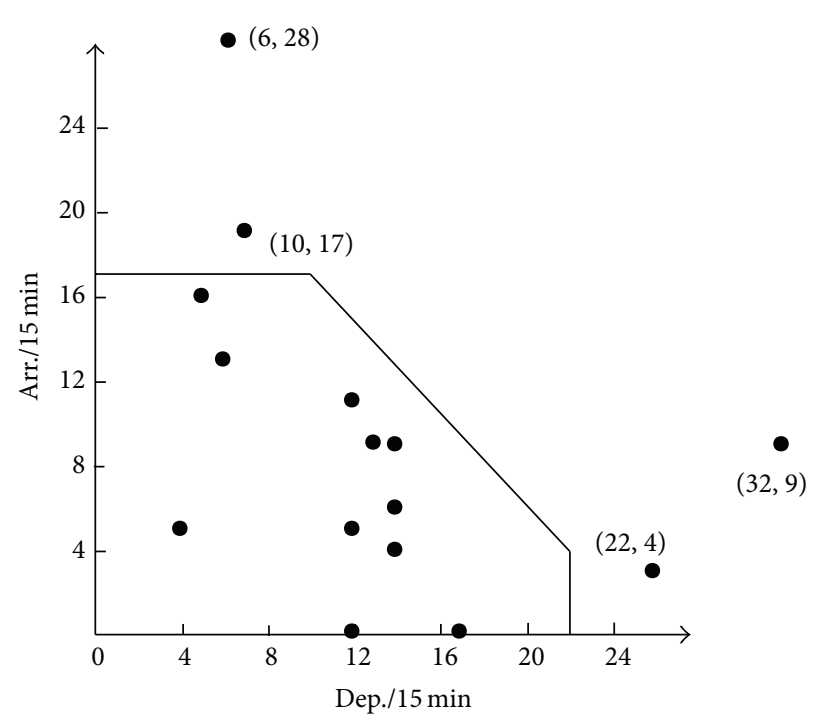

(b) Shanghai PuDong Airport

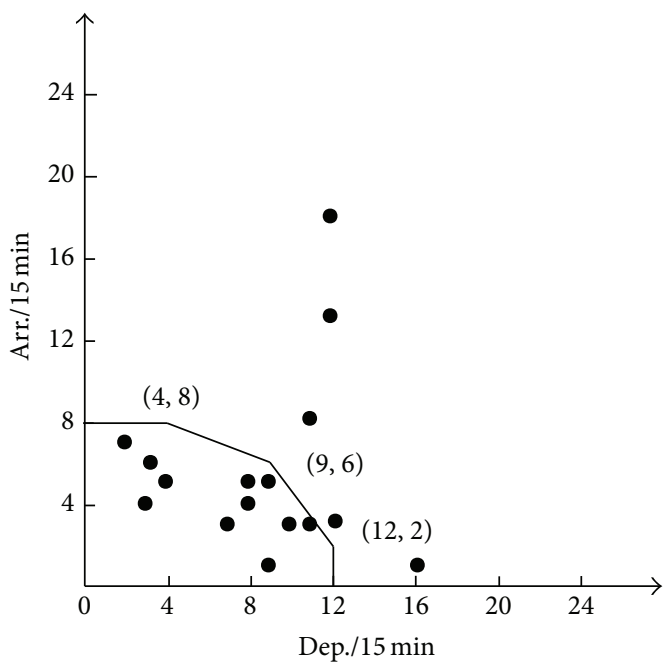

(c) Guangzhou BaiYun Airport

FIGURE 3: Match charts of flow and capacity in the three airports before optimization.

figure), and an imbalanced demand and supply will naturally result in flight delay.

5.2. Model Optimization Analysis. Airport flow assignment problem is essential to reassign times of arrival or departure of affected flights with some constraints; it belongs to combinatorial optimization problem. The model is $0-1$ integer programming model. There are many optimization methods to solve it. Because the model can deal with a lot of flights and airports in an open network, and the actual decisionmaking needs to address the balance between supply and demand during several hours, involving a large amount of data, a method suitable for solving large-scale integer programming problem should be used; we use LINGO software for the model simulation, and set $\delta=0.01$. Flow distribution results of each period and the matching conditions of flow and capacity in the three airports after optimization are shown in Table 2 and Figure 4; the total delays time is 3060 minutes. Apparently, Figure 4 shows that the optimized points in all traffic are distributed within or on the capacity curve, which indicates that traffic flow has been coordinated with the capacity. Figure 5 compares the traffic flow before and after optimization in the three airports and shows the optimized traffic distribution scheme, which not only effectively eliminates the traffic peak, but also balances the flow distribution among those times, takes 
TABLE 2: The flow distribution in the three airports after optimization.

\begin{tabular}{|c|c|c|c|c|c|c|c|c|c|}
\hline \multirow{2}{*}{ Time } & \multicolumn{3}{|c|}{ Beijing Capital Airport } & \multicolumn{3}{|c|}{ Shanghai PuDong Airport } & \multicolumn{3}{|c|}{ Guangzhou BaiYun Airport } \\
\hline & Dep. & Arr. & Total & Dep. & Arr. & Total & Dep. & Arr. & Total \\
\hline $8: 00-8: 14$ & 14 & 0 & 14 & 12 & 0 & 12 & 12 & 1 & 13 \\
\hline $8: 15-8: 29$ & 17 & 1 & 18 & 17 & 0 & 17 & 12 & 2 & 14 \\
\hline $8: 30-8: 44$ & 16 & 3 & 19 & 14 & 6 & 20 & 12 & 2 & 14 \\
\hline $8: 45-8: 59$ & 15 & 9 & 24 & 14 & 4 & 18 & 9 & 5 & 14 \\
\hline 9:00-9:14 & 7 & 4 & 11 & 22 & 3 & 25 & 11 & 3 & 14 \\
\hline $9: 15-9: 29$ & 5 & 2 & 7 & 15 & 5 & 20 & 4 & 5 & 9 \\
\hline $9: 30-9: 44$ & 10 & 13 & 23 & 4 & 5 & 9 & 2 & 7 & 9 \\
\hline 9:45-9:59 & 8 & 15 & 23 & 10 & 17 & 27 & 9 & 6 & 15 \\
\hline 10:00-10:14 & 5 & 6 & 11 & 10 & 17 & 27 & 9 & 6 & 15 \\
\hline $10: 15-10: 29$ & 1 & 5 & 6 & 10 & 17 & 27 & 4 & 4 & 8 \\
\hline $10: 30-10: 44$ & 16 & 8 & 24 & 8 & 17 & 25 & 10 & 3 & 13 \\
\hline $10: 45-10: 59$ & 9 & 15 & 24 & 8 & 17 & 25 & 9 & 6 & 15 \\
\hline 11:00-11:14 & 12 & 12 & 24 & 10 & 17 & 27 & 9 & 6 & 15 \\
\hline $11: 15-11: 29$ & 8 & 15 & 23 & 10 & 17 & 27 & 4 & 8 & 12 \\
\hline 11:30-11:44 & 10 & 8 & 18 & 10 & 17 & 27 & 9 & 6 & 15 \\
\hline $11: 45-12: 00$ & 11 & 13 & 24 & 21 & 5 & 26 & 9 & 6 & 15 \\
\hline $12: 00$ & 10 & 7 & 17 & 11 & 11 & 22 & 3 & 13 & 16 \\
\hline Total & 174 & 136 & 310 & 206 & 175 & 381 & 137 & 89 & 226 \\
\hline
\end{tabular}

TABLE 3: Optimization results of different parameters.

\begin{tabular}{lccc}
\hline$\delta$ & $\begin{array}{r}\text { Total delay } \\
\text { time (min) }\end{array}$ & $\begin{array}{c}\text { Standard deviation } \\
\text { of airline delay }\end{array}$ & $\begin{array}{c}\text { Optimization } \\
\text { duration (sec) }\end{array}$ \\
\hline 0.001 & 3077 & 0.005567 & 181 \\
0.002 & 3075 & 0.061058 & 55 \\
0.005 & 3072 & 0.074080 & 56 \\
0.008 & 3060 & 0.072691 & 48 \\
0.010 & 3060 & 0.097182 & 14 \\
0.050 & 3060 & 0.928612 & 16 \\
0.200 & 3060 & 0.897701 & 11 \\
0.500 & 3060 & 0.864192 & 11 \\
0.800 & 3060 & 1.124511 & 10 \\
1.000 & 3060 & 1.328926 & 11 \\
\hline
\end{tabular}

full advantage of the limited capacities of these airports, and gives reasonable arrangements for the flights to take off or land.

Figure 6 shows that airlines share delay loss caused by allocation. We can see that the average flight delays of airlines are close to $3.3 \mathrm{~min}$, in which is less than $0.3 \mathrm{~min}$, so the delay is relatively balanced; those effectiveness-fair degrees of airlines are between 0.98 and 1.06 and are closed to 1, so the delay is relatively fair. For example, the effectiveness-fair degrees of airlines 1 and 2 are both equal to 1 , which is shown to be absolutely fair to them.

5.3. Parameter Affects Analysis. In order to analyze the impact of the parameter $\delta$, we selected 10 cases to optimize. The performance and the optimization results are shown in Table 3; the analysis results of average delay and effectivenessfair degree of airlines are shown in Figure 7. It can be seen from Table 3 that, with the increase of $\delta$, the total delay time 


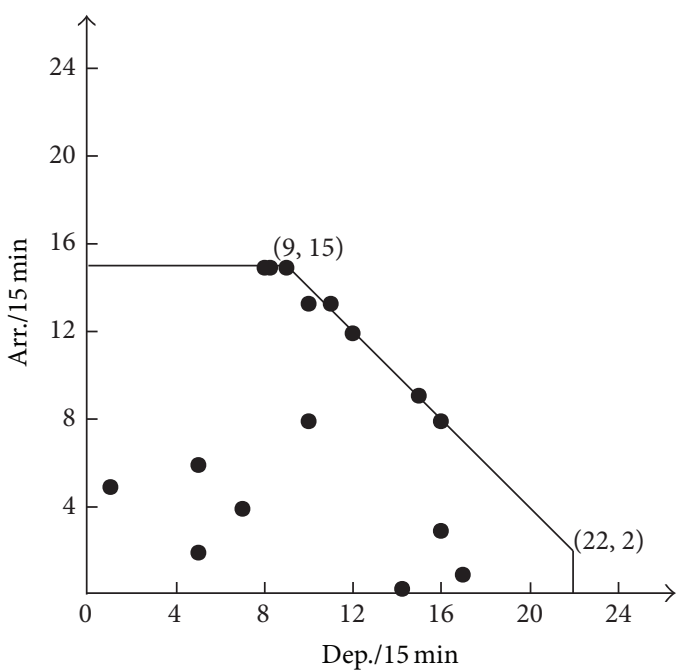

(a) Beijing Capital Airport

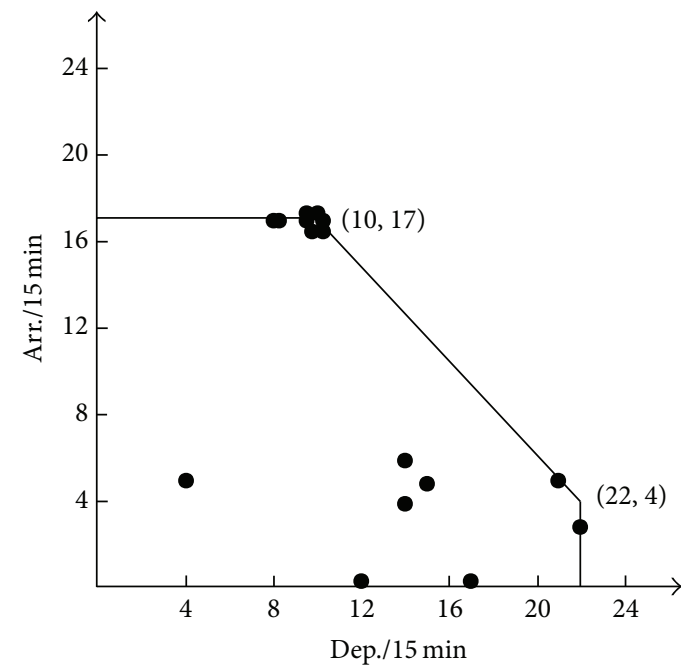

(b) Shanghai PuDong Airport

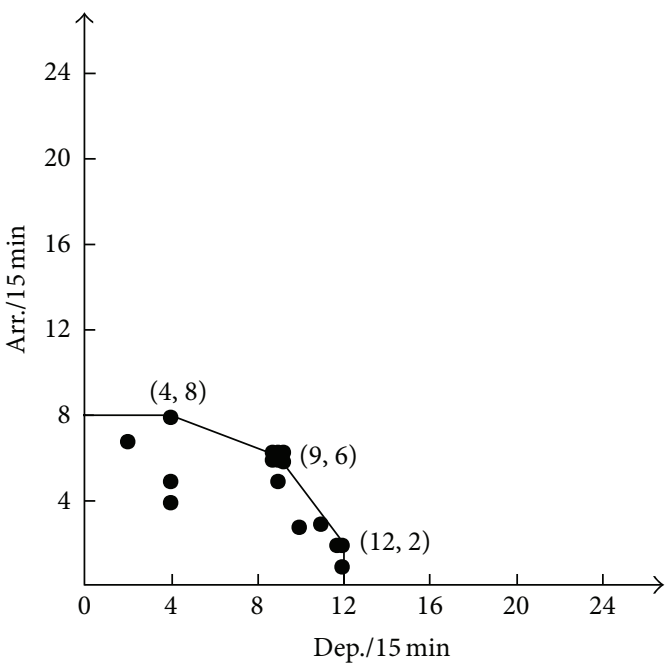

(c) Guangzhou BaiYun Airport

FIGURE 4: Match charts of flow and capacity in the three airports after optimization.

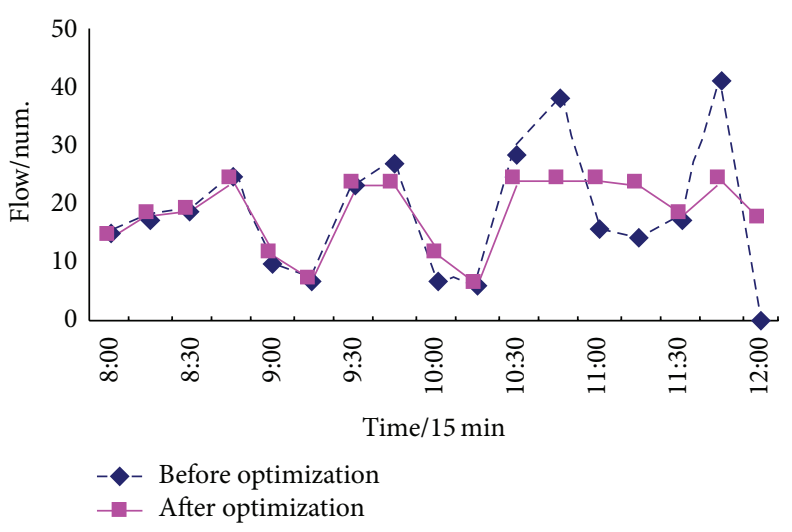

(a) Beijing Capital Airport

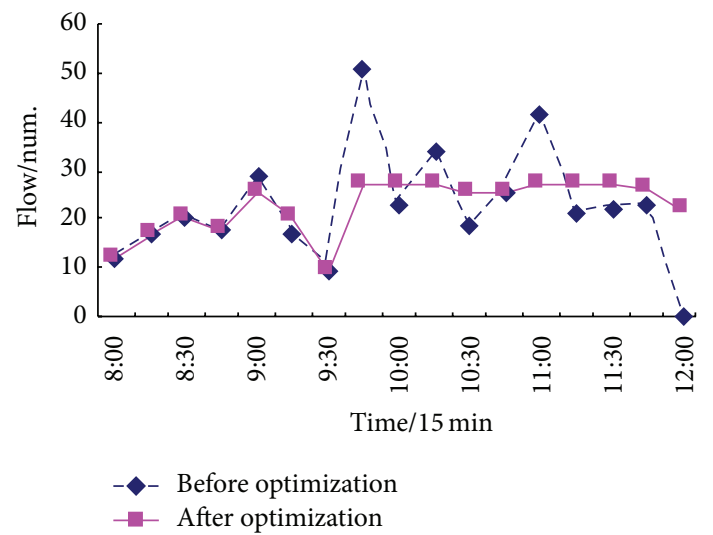

(b) Shanghai PuDong Airport

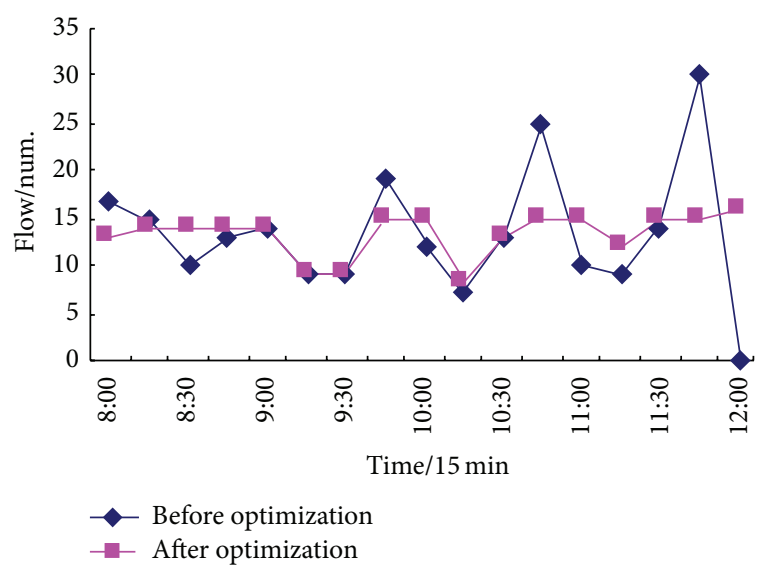

(c) Guangzhou BaiYun Airport

Figure 5: Traffic flows in three airports before and after optimization.

is declining and the effectiveness is gradually increasing, but when it increases to a certain extent, it tends to a constant. The increasing of standard deviation of delay reflects the reduction of fairness. The optimization duration tends to decrease, indicating that a more relaxed constraint makes it easy to obtain the optimal solution. While the value of $\delta$ is smaller $(\delta \leq 0.08)$, the fairness and effectiveness are a tradeoff, basically conflicting. In contrast, while the value of 

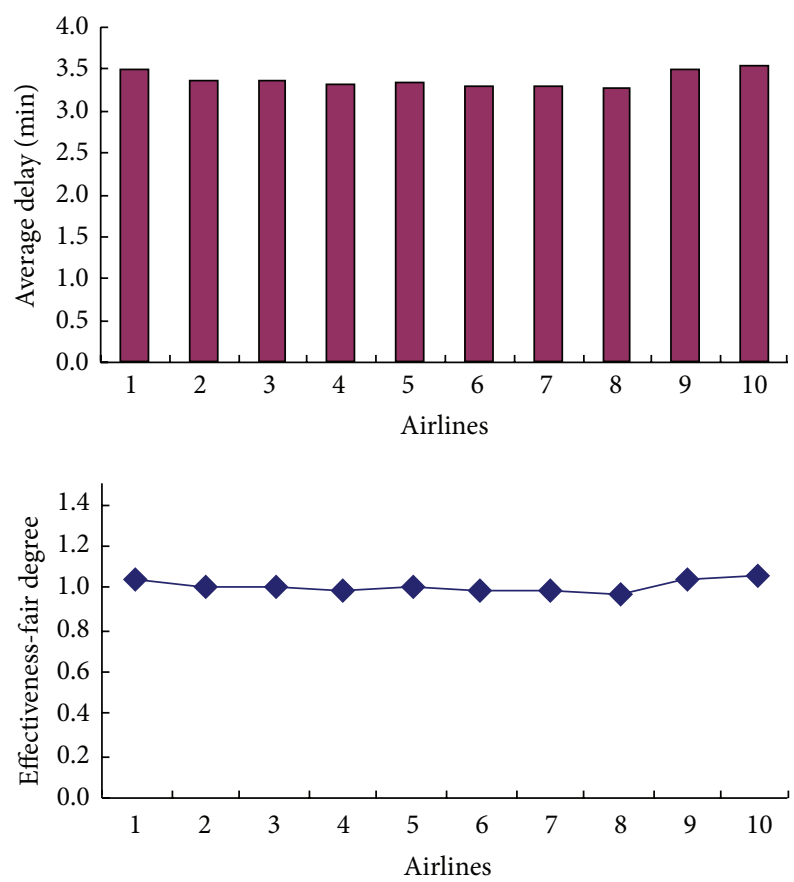

FIGURE 6: Analysis of user equilibrium.

$\delta$ is larger $(0.08<\delta \leq 1)$, the total delay tends to a constant, which no longer affects the effectiveness.

In Figure 7 , with the decrease of $\delta$, the change of average delay of airlines is leveling off and effectiveness-fair degrees trend to be 1 . It means that the deviation of the average delay between airlines is continuing to decline and the injustice input on the single airline has also been reduced; that is to say, fairness continues to increase. Therefore, the selection of $\delta$ value has an important influence on the optimization results. In accordance with the provisions of United Nations organizations, the Gini coefficient which is between 0.2 and 0.3 shows balance. Considering effectiveness, fairness, and efficiency factors, we found that the $\delta$ values between 0.002 and 0.01 are better.

\section{Conclusions}

(1) A user equilibrium assignment flow model for multiairport open network system is proposed in this paper; it can be used to balance supply and demand in airport network system with the view of both the system and users.

(2) The test results show that the model can optimize the matching relationship of flow and capacity in a multiairport open network system and make full use of the capacity of airports and minimize the system delays, so that it can provide a powerful reference of developing scientific and rational assignment strategy for air traffic controllers.

(3) If the model is extended to the whole air traffic network, which consists of airports, sectors, en-route, and so forth, further studies on the problem of demand and supply imbalance in more complicated large-scale regional air traffic can be carried on.
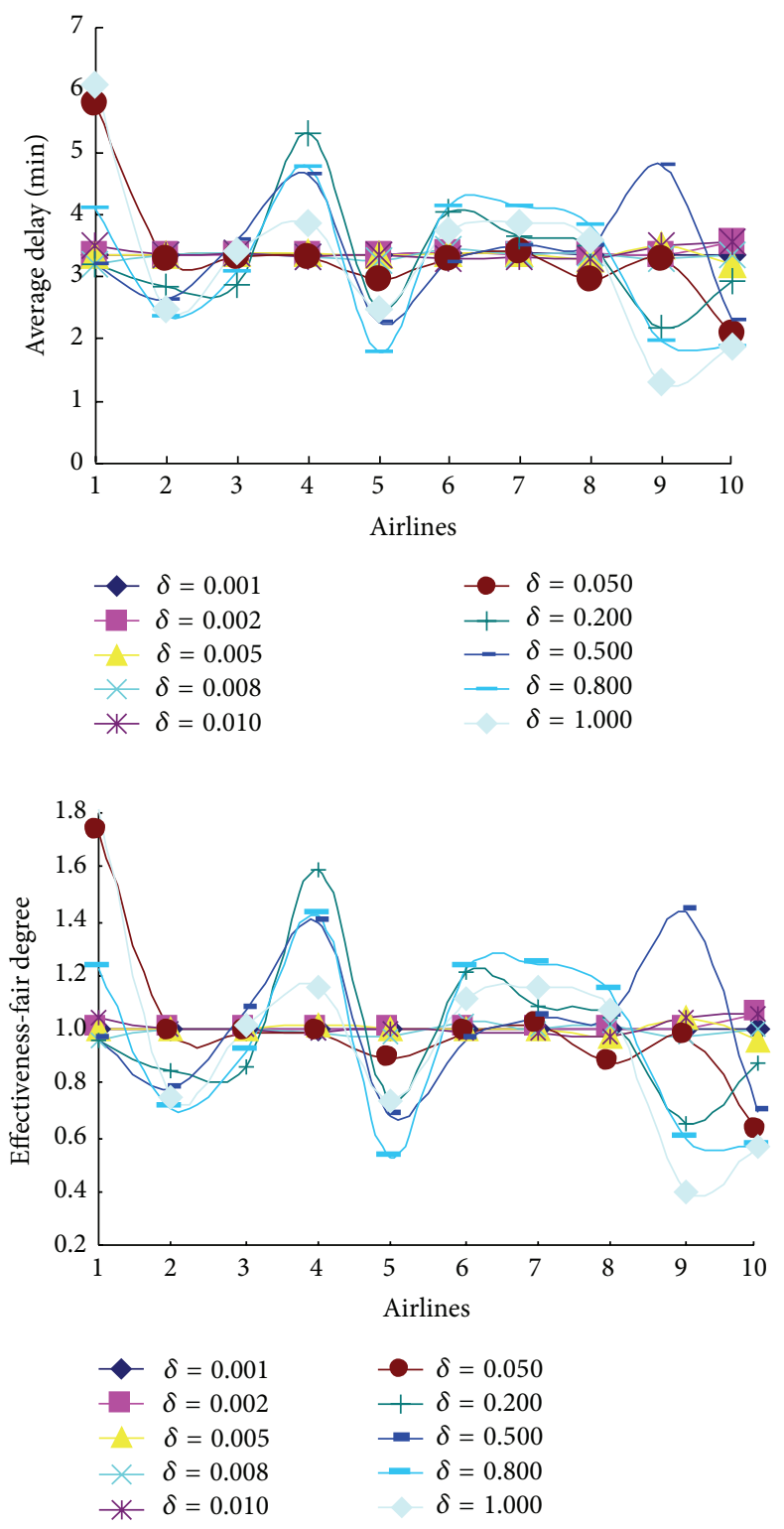

FIgURE 7: Analysis of user equilibrium of different parameters.

\section{Conflict of Interests}

The authors declare that there is no conflict of interests regarding the publication of this paper.

\section{Acknowledgment}

This study is funded by the Fundamental Research Funds for the Central University (NS2014068).

\section{References}

[1] FAA, "1993 aviation system capacity plan," Tech. Rep. DOT/FAA/ASC-93-1, Office of System Capacity and Requirements, Washington, DC, USA, 1993. 
[2] S. G. Hamzawi, "Lack of airport capacity: exploration of alternative solutions," Transportation Research Part A, vol. 26, no. 1, pp. 47-58, 1992.

[3] Airport Association, Airport/Airway System Capacity Needs, Airports Council International-North America, Washington, DC, USA, 1992.

[4] H. H. Zhang, The key technologies of collaborative flow management in airport terminal area [Ph.D. thesis], Nanjing University of Aeronautics and Astronautics, Nanjing, China, 2009, (Chinese).

[5] Z. P. Ma, D. G. Cui, and Y. L. Xie, "Flow distribution and optimal scheduling in airport terminal area," Journal of Tsinghua University (Science and Technology), vol. 43, no. 7, pp. 876-879, 2003 (Chinese).

[6] X. Chen, X. Lu, and J. Zhu, "Optimization model of hubairport airside capacity utilization and flow allocation," Journal of Nanjing University of Aeronautics and Astronautics, vol. 39, no. 5, pp. 680-684, 2007.

[7] P. Dell'Olmo and G. Lulli, "A dynamic programming approach for the airport capacity allocation problem," IMA Journal of Management Mathematics, vol. 14, no. 3, pp. 235-249, 2003.

[8] H. Zhang, M. Hu, and S. Chen, "Collaborative distribution strategy of airport arrival and departure traffic flow," Journal of Nanjing University of Aeronautics and Astronautics, vol. 40, no. 5, pp. 641-645, 2008 (Chinese).

[9] H. Zhang, M. Hu, and S. Chen, "Collaborative optimization of capacity utilization and flow assignment in airport terminal area," Journal of Southwest Jiaotong University, vol. 44, no. 1, pp. 128-134, 2009.

[10] H.-H. Zhang and M.-H. Hu, "Modeling and simulation of capacity utilization and flow assignment in multi-airport terminal area," Journal of System Simulation, vol. 21, no. 18, pp. 56395644, 2009 (Chinese).

[11] Y. Tian, K. Song, and Y. H. Gu, "The research on rerouting problem in air traffic flow management," Mathematics in Practice and Theory, vol. 38, no. 10, pp. 70-76, 2008 (Chinese).

[12] E. P. Gilbo, "Optimizing airport capacity utilization in air traffic flow management subject to constraints at arrival and departure fixes," IEEE Transactions on Control Systems Technology, vol. 5, no. 5, pp. 490-503, 1997.

[13] E. P. Gilbo, "Airport capacity: representation, estimation, optimization," IEEE Transactions on Control Systems Technology, vol. 1, no. 3, pp. 144-154, 1993. 


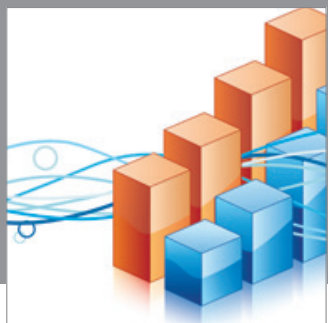

Advances in

Operations Research

mansans

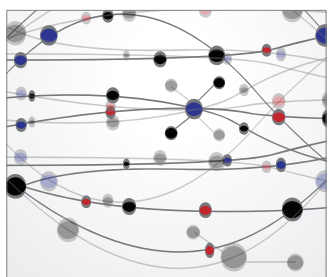

The Scientific World Journal
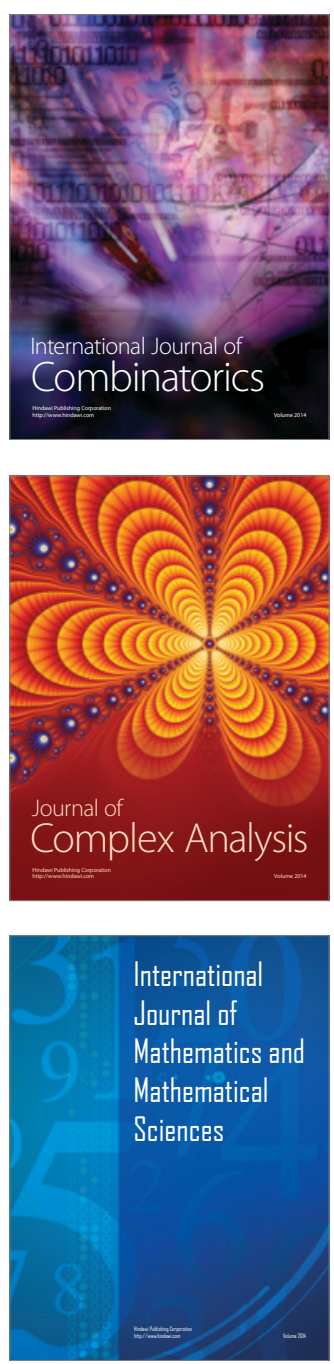
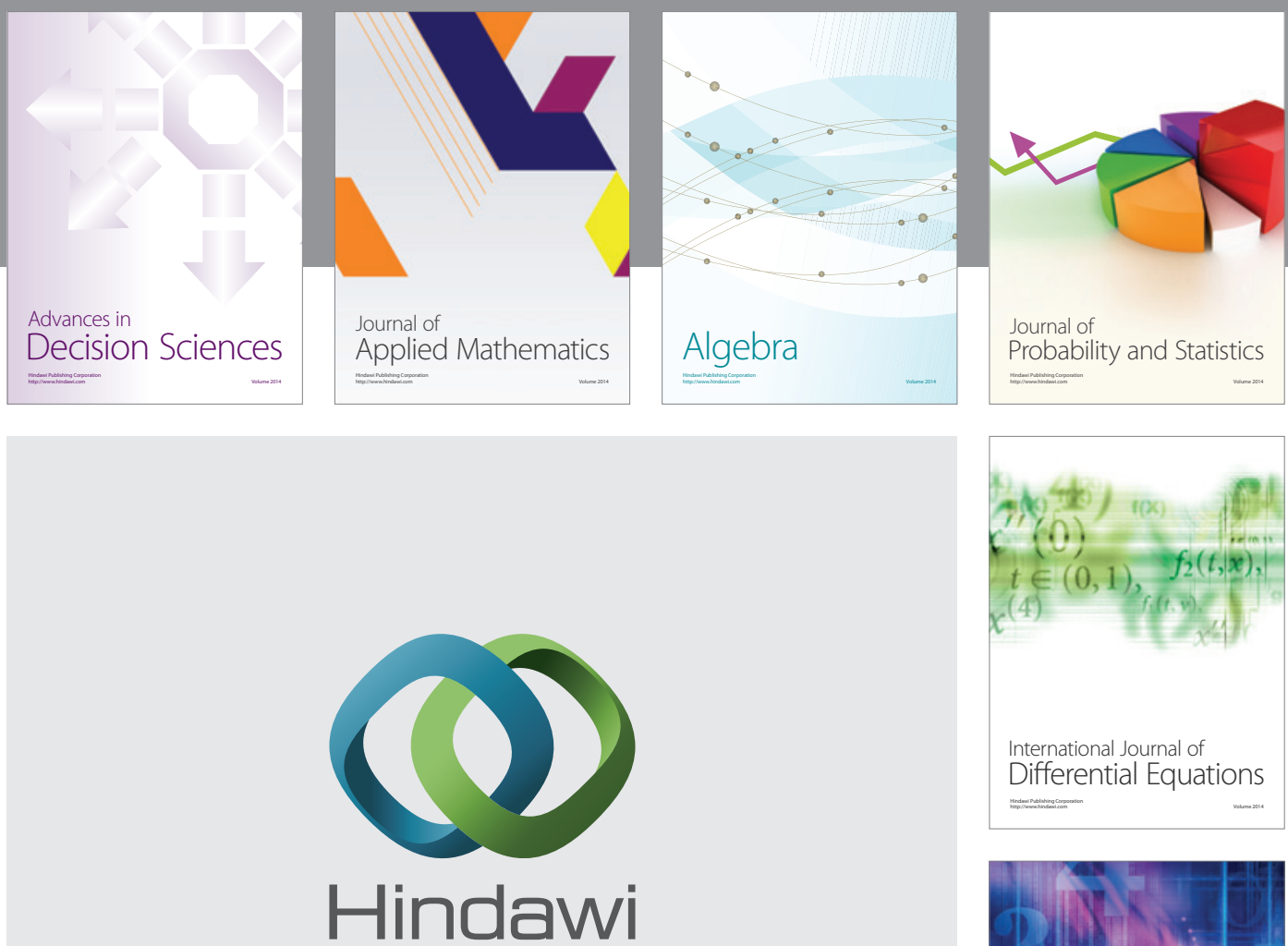

Submit your manuscripts at http://www.hindawi.com
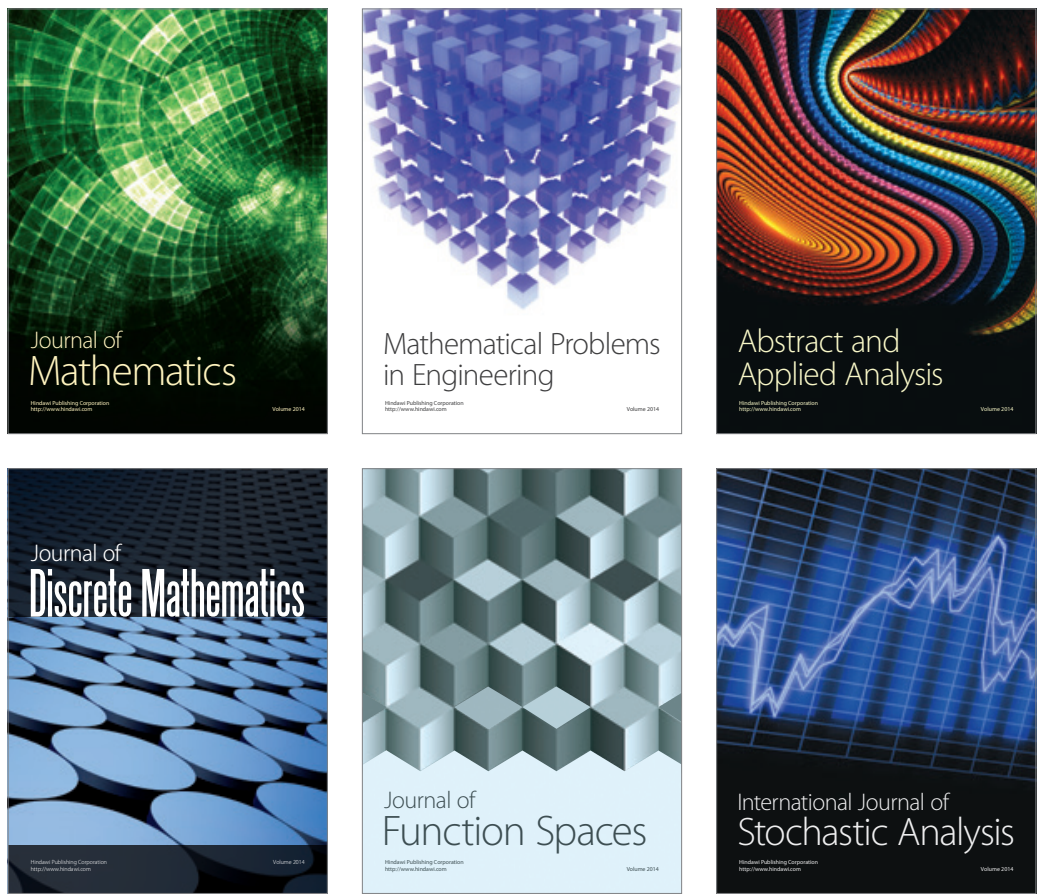

Journal of

Function Spaces

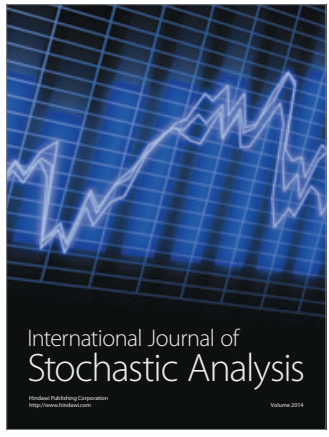

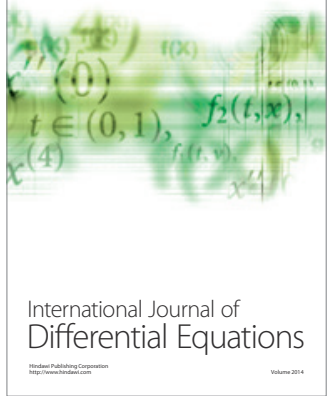
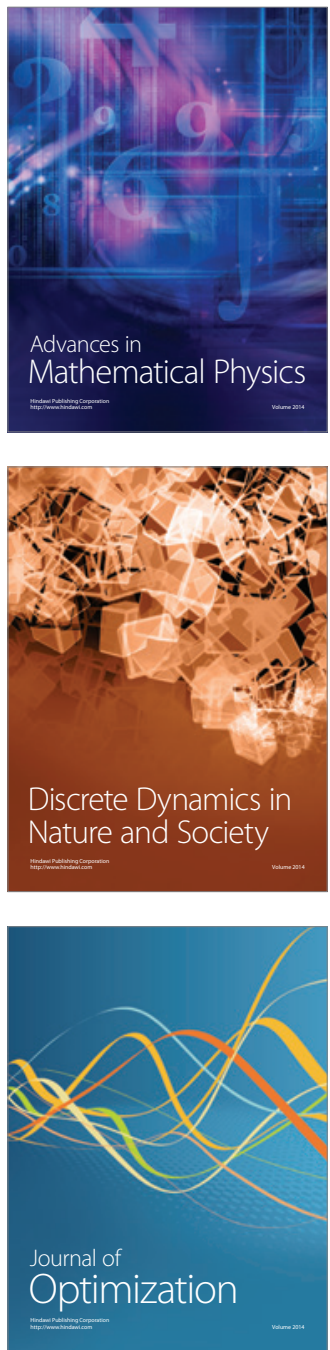